\title{
Multiplicity of solutions of perturbed Schrödinger equation with electromagnetic fields and critical nonlinearity in $\mathbb{R}^{N}$
}

Sihua Liang ${ }^{1,2}$ and Yueqiang Song ${ }^{3^{*}}$

*Correspondence:

songyueqiang@sohu.com ${ }^{3}$ Scientific Research Department,

Changchun Normal University, Changchun, Jilin 130032, P.R. China Full list of author information is available at the end of the article

\begin{abstract}
In this paper, we deal with the existence and multiplicity of solutions for perturbed Schrödinger equation with electromagnetic fields and critical nonlinearity in $\mathbb{R}^{N}$ : $-\varepsilon^{2} \Delta_{A} u(x)+V(x) u(x)=|u|^{2^{*}-2} u+h\left(x,|u|^{2}\right) u$ for all $x \in \mathbb{R}^{N}$, where $\nabla_{A} u(x):=(\nabla+i A(x)) u$, $V(x)$ is a nonnegative potential. By using Lions' second concentration compactness principle and concentration compactness principle at infinity to prove that the $(P S)_{c}$ condition holds locally and by variational method, we show that this equation has at least one solution provided that $\varepsilon<\mathcal{E}$, for any $m \in \mathbb{N}$, it has $m$ pairs of solutions if $\varepsilon<\mathcal{E}_{m}$, where $\mathcal{E}$ and $\mathcal{E}_{m}$ are sufficiently small positive numbers.
\end{abstract}

MSC: $35 \mathrm{~J} 60 ; 35 \mathrm{~B} 33$

Keywords: perturbed Schrödinger equation; critical nonlinearity; magnetic fields; variational methods

\section{Introduction}

In this paper, we are concerned with the existence of nontrivial solutions to the following perturbed Schrödinger equation with electromagnetic fields and critical nonlinearity in $\mathbb{R}^{N}$ :

$$
-\varepsilon^{2} \Delta_{A} u(x)+V(x) u(x)=|u|^{2^{*}-2} u+h\left(x,|u|^{2}\right) u \quad \text { for } x \in \mathbb{R}^{N},
$$

where $\nabla_{A} u(x):=(\nabla+i A(x)) u$. Here, $i$ is the imaginary unit, $2^{*}:=2 N /(N-2)$ denotes the Sobolev critical exponent and $N \geq 3, V(x)$ and $h(x, u)$ are functions satisfying some conditions.

This paper is motivated by some works concerning the nonlinear Schrödinger equation

$$
\begin{aligned}
i \hbar \frac{\partial \psi}{\partial t}= & -\frac{\hbar^{2}}{2 m}(\nabla+i A(x))^{2} \psi+W(x) \psi \\
& -K(x)|\psi|^{2^{*}-2} \psi-h\left(x,|\psi|^{2}\right) \psi \quad \text { for } x \in \mathbb{R}^{N},
\end{aligned}
$$

where $\hbar$ is Plank constant, $A(x)=\left(A_{1}(x), A_{2}(x), \ldots, A_{N}(x)\right): \mathbb{R}^{N} \rightarrow \mathbb{R}^{N}$ is a real vector (magnetic) potential with magnetic field $B=\operatorname{curl} A$ and $W(x): \mathbb{R}^{N} \rightarrow \mathbb{R}^{N}$ is a scalar electric potential.

( 2014 Liang and Song; licensee Springer. This is an Open Access article distributed under the terms of the Creative Commons Attribution License (http://creativecommons.org/licenses/by/4.0), which permits unrestricted use, distribution, and reproduction in any medium, provided the original work is properly credited. 
In physics, we are interested in standing wave solutions, i.e., solutions of type (1.2) when $\hbar$ is sufficiently small, when $E$ is a real number and $u(x)$ is a complex-value function which satisfies

$$
-(\nabla+i A(x))^{2} u(x)+\lambda V(x) u(x)=\lambda K(x)|u|^{2^{*}-2} u+\lambda h\left(x,|u|^{2}\right) u, \quad x \in \mathbb{R}^{N},
$$

where $\lambda^{-1}=\frac{\hbar^{2}}{2 m}$ and $V(x)=W(x)-E$. The transition from quantum mechanics to classical mechanics can be formally performed by letting $\hbar \rightarrow 0$. Thus the existence of solutions for $\hbar$ small, semi-classical solutions, has important physical interest.

It is well known that the linear Schrödinger equation is a basic tool of quantum mechanics, and it provides a description of the dynamics of a particle in a non-relativistic setting. The nonlinear Schrödinger equation arises in different physical theories, e.g., the description of Bose-Einstein condensates and nonlinear optics, see [1] and the references cited there. Both the linear and the nonlinear Schrödinger equations have been widely considered in the literature. The main purpose of this paper is to study the existence and multiplicity of solutions of perturbed Schrödinger equations with electromagnetic fields and critical nonlinearity (1.1).

Problem (1.3) with $A(x) \equiv 0$ has an extensive literature. Different approaches have been taken to attack this problem under various hypotheses on the potential and the nonlinearity. See, for example, [2-13] and the references therein. Observe that in all these papers the nonlinearities are assumed to be subcritical. In [11], using a Lyapunov-Schmidt reduction, Floer and Weinstein established the existence of single and multiple spike solutions. Their method and results were later generalized by $\mathrm{Oh}$ [12] to the higher-dimensional case. Kang and Wei [14] established the existence of positive solutions with any prescribed number of spikes, clustering around a given local maximum point of the potential function. In accordance with the Sobolev critical nonlinearities, there have been many papers devoted to studying the existence of solutions to elliptic boundary-valued problems on bounded domains after the pioneering work by Brezis and Nirenberg [15]. Ding and Lin [9] first studied the existence of semi-classical solutions to the problem on the whole space with critical nonlinearities and established the existence of positive solutions as well as of those that change sign exactly once. They also obtained multiplicity of solutions when the nonlinearity is odd.

When $A(x) \not \equiv 0$, there are also many works dealing with the magnetic case. The first one seems to be [16] where the existence of standing waves was obtained for $\hbar>0$ fixed and for special classes of magnetic fields. If $A$ and $W$ are periodic functions, the existence of various types of solutions for fixed $\hbar>0$ was proved in [17] by applying minimax arguments. Concerning semi-classical bound states, it was proved in [18] that for $\hbar>0$ small and admits a least energy solution which concentrates near the global minimum of $W$. A multiplicity result for solutions was obtained in [4] by using a topological argument. There it was also proved that the magnetic potential $A$ only contributes to the phase factor of the solitary solutions for $\hbar>0$ sufficiently small. In [19] single-bump bound states were obtained by using perturbation methods. These concentrate near a non-degenerate critical point of $W$ as $\hbar \rightarrow 0$. For the critical growth case, Wang [20] studied the electromagnetic Schrödinger equations

$$
-(\nabla+i A(x))^{2} u(x)+\lambda V(x) u(x)=K(x)|u|^{2^{*}-2} u \quad \text { for } x \in \mathbb{R}^{N} .
$$


By using the linking theorem twice to the corresponding functional, they established the existence results. Chabrowski and Szulkin [21] considered problem (1.4) under assumption that $V(x)$ changes sign; by using a min-max type argument based on a topological linking, they obtained a solution in the Sobolev space which was defined in the paper. Assume $K(x) \equiv 1$, Han [22] studied problem (1.4) and established the existence of nontrivial solutions in the critical case by means of variational method. For more results, we refer the reader to [20,23-27] and the references therein.

In the present paper, we consider the existence of solutions for problem (1.1) under the condition $\inf _{x \in \mathbb{R}^{N}} V(x)=0$ and critical nonlinearity. It seems that Byeon and Wang [1] were the first to study energy level and the asymptotic behavior of positive solutions to Schrödinger equations under the condition $\inf _{x \in \mathbb{R}^{N}} V(x)=0$. In [25], Cao and Tang extended the results of Byeon and Wang [1]. However, to the best knowledge, it seems that there are few works on the existence of solutions to be the problems on $\mathbb{R}^{N}$ involving critical nonlinearities with electromagnetic fields. We mainly follow the idea of $[9,10]$. Let us point out that although the idea was used before for other problems, the adaptation of the procedure to our problem is not trivial at all. Because of the appearance of electromagnetic potential $A(x)$, we must consider our problem for complex-valued functions, and so we need more delicate estimates. Furthermore, we use Lions' second concentration compactness principle and concentration compactness principle at infinity to prove that the $(P S)_{c}$ condition holds, which is different from methods used in $[9,10]$.

\section{Main results}

Let $\lambda=\varepsilon^{-2}$. Equation (1.1) reads then as

$$
-(\nabla+i A(x))^{2} u(x)+\lambda V(x) u(x)=\lambda|u|^{2^{*}-2} u+\lambda h\left(x,|u|^{2}\right) u \quad \text { for } x \in \mathbb{R}^{N}
$$

We make the following assumptions on $A(x), V(x)$ and $h$ throughout this paper:

(V) $V(x) \in C\left(\mathbb{R}^{N}, \mathbb{R}\right) ; V\left(x_{0}\right)=\min _{x \in \mathbb{R}^{N}} V=0$, and there is $b>0$ such that the set $V^{b}=\left\{x \in \mathbb{R}^{N}: V(x)<b\right\}$ has finite Lebesgue measure;

(A) $A_{j}(x) \in C\left(\mathbb{R}^{N}, \mathbb{R}\right)(j=1,2, \ldots, N)$ and $A\left(x_{0}\right)=0$;

(H) $\left(\mathrm{h}_{1}\right) h \in C\left(\mathbb{R}^{N} \times[0,+\infty), \mathbb{R}\right)$ and $h(x, t)=o(1)$ uniformly in $x$ as $t \rightarrow 0$;

$\left(\mathrm{h}_{2}\right)$ there are $C_{0}>0$ and $q \in\left(2,2^{*}\right)$ such that $|h(x, t)| \leq C_{0}\left(1+t^{\frac{q-2}{2}}\right)$;

$\left(\mathrm{h}_{3}\right)$ there are $a_{0}>0, p>2$ and $\mu>2$ such that $H(x, t) \geq a_{0} t^{\frac{p}{2}}$ and $\frac{\mu}{2} H(x, t) \leq h(x, t) t$ for all $(x, t)$, where $H(x, t)=\int_{0}^{t} h(x, s) d s$.

Set

$$
\nabla_{A} u=(\nabla+i A(x)) u
$$

and

$$
H_{A}^{1}\left(\mathbb{R}^{N}\right)=\left\{u \in L^{2}\left(\mathbb{R}^{N}\right): \nabla_{A} u \in L^{2}\left(\mathbb{R}^{N}\right)\right\}
$$

Hence $H_{A}^{1}\left(\mathbb{R}^{N}\right)$ is the Hilbert space under the scalar product

$$
\langle u, v\rangle=\operatorname{Re} \int_{\mathbb{R}^{N}}((\nabla u+i A(x) u) \overline{(\nabla v+i A(x) v)}+u \bar{v}) d x,
$$


the norm induced by the product $(\cdot, \cdot)$ is

$$
\begin{aligned}
\|u\|_{H_{A}^{1}\left(\mathbb{R}^{N}\right)} & =\left(\int_{\mathbb{R}^{N}}\left(\left|\nabla \nabla_{A} u\right|^{2}+|u|^{2}\right) d x\right)^{\frac{1}{2}} \\
& =\left(\int_{\mathbb{R}^{N}}\left(|\nabla u+i A(x) u|^{2}+|u|^{2}\right) d x\right)^{\frac{1}{2}} \\
& =\left(\int_{\mathbb{R}^{N}}\left(|\nabla u|^{2}+\left(|i A(x)|^{2}+1\right)|u|^{2}\right) d x-2 \operatorname{Re} \int_{\mathbb{R}^{N}} i A(x) \bar{u} \nabla u d x\right)^{\frac{1}{2}} .
\end{aligned}
$$

Let

$$
E:=\left\{u \in H_{A}^{1}\left(\mathbb{R}^{N}\right): \int_{\mathbb{R}^{N}} \lambda V(x)|u|^{2} d x<\infty\right\}
$$

which is a Hilbert space equipped with the norm

$$
\|u\|_{\lambda}^{2}=\int_{\mathbb{R}^{N}}\left(\left|\nabla_{A} u\right|^{2}+\lambda V(x)|u|^{2}\right) d x .
$$

Remark 2.1 We have the following diamagnetic inequality (see [16] for example):

$$
\left|\nabla_{A} u(x)\right| \geq|\nabla| u(x)|| \quad \text { for } u \in H_{A}^{1}\left(\mathbb{R}^{N}\right) .
$$

Indeed, since $A$ is real-valued,

$$
|\nabla| u|(x)|=\left|\operatorname{Re}\left(\nabla u \frac{\bar{u}}{|u|}\right)\right|=\left|\operatorname{Re}(\nabla u+i A u) \frac{\bar{u}}{|u|}\right| \leq|\nabla u+i A u|
$$

(the bar denotes complex conjugation) this fact means that if $u \in H_{A}^{1}\left(\mathbb{R}^{N}\right)$, then $|u| \in$ $H^{1}\left(\mathbb{R}^{N}\right)$, and therefore $u \in L^{p}\left(\mathbb{R}^{N}\right)$ for any $p \in\left[2,2^{*}\right]$.

Remark 2.2 The spaces $H_{A}^{1}\left(\mathbb{R}^{N}\right)$ and the spaces $H^{1}\left(\mathbb{R}^{N}\right)$ are not comparable; more precisely, in general $H_{A}^{1}\left(\mathbb{R}^{N}\right) \nsubseteq H^{1}\left(\mathbb{R}^{N}\right)$ and $H^{1}\left(\mathbb{R}^{N}\right) \nsubseteq H_{A}^{1}\left(\mathbb{R}^{N}\right)$. However, it is proved by Arioli and Szulkin [17] that if $K$ is a bounded domain with regular boundary, then $H_{A}^{1}\left(\mathbb{R}^{N}\right)$ and $H^{1}\left(\mathbb{R}^{N}\right)$ are equivalent, where $H_{A}^{1}(K)=\left\{u \in L^{2}(K): \nabla u \in L^{2}(K)\right\}$ with the norm $\|u\|_{H_{A}^{1}(K)}=\left(\int_{K}\left(\left|\nabla_{A} u\right|^{2}+|u|^{2}\right) d x\right)^{\frac{1}{2}}$.

Let

$$
E_{\lambda}:=\left\{u \in H_{A}^{1}\left(\mathbb{R}^{N}\right): \int_{\mathbb{R}^{N}} V(x)|u|^{2} d x<\infty\right\}
$$

with the norms

$$
\|u\|_{\lambda}^{2}=\int_{\mathbb{R}^{N}}\left(\left|\nabla_{A} u\right|^{2}+\lambda V(x)|u|^{2}\right) d x .
$$

Thus, it is easy to see that the norm $\|\cdot\|_{E}$ is equivalent to the one $\|\cdot\|_{\lambda}$ for each $\lambda>0$. From Remark 2.2, for each $s \in\left[2,2^{*}\right]$, there is $c_{s}>0$ (independent of $\lambda$ ) such that if $\lambda>1$, 
then

$$
\left(\int_{\mathbb{R}^{N}}|u|^{s}\right)^{\frac{1}{s}} \leq c_{s}\left(\int_{\mathbb{R}^{N}}|\nabla| u||^{2}\right)^{\frac{1}{2}} \leq c_{s}\left(\int_{\mathbb{R}^{N}}\left|\nabla_{A} u\right|^{2}\right)^{\frac{1}{2}} \leq c_{s}\|u\|_{\lambda} .
$$

Consider the functional

$$
\begin{aligned}
J_{\lambda}(u):= & \frac{1}{2} \int_{\mathbb{R}^{N}}\left(\left|\nabla_{A} u\right|^{2}+\lambda V(x)|u|^{2}\right) d x-\frac{\lambda}{2^{*}} \int_{\mathbb{R}^{N}}|u|^{2^{*}} d x \\
& -\frac{\lambda}{2} \int_{\mathbb{R}^{N}} H\left(x,|u|^{2}\right) d x \\
= & \frac{1}{2}\|u\|_{\lambda}^{2}-\frac{\lambda}{2^{*}} \int_{\mathbb{R}^{N}}|u|^{2^{*}} d x-\frac{\lambda}{2} \int_{\mathbb{R}^{N}} H\left(x,|u|^{2}\right) d x .
\end{aligned}
$$

Under the assumptions [28], $J_{\lambda} \in C^{1}\left(E_{\lambda}, \mathbb{R}\right)$ and its critical points are solutions of (2.1).

Theorem 2.1 Let (V), (A) and (H) be satisfied. Thus:

(1) For any $\sigma>0$, there is $\Lambda_{\sigma}>0$ such that problem (2.1) has at least one solution $u_{\lambda}$ for each $\lambda \geq \Lambda_{\sigma}$ satisfying $0<J_{\lambda}\left(u_{\lambda}\right) \leq \sigma \lambda^{1-\frac{N}{2}}$.

(2) Assume additionally that $h(x, t)$ is odd in $t$; for any $m \in N$ and $\sigma>0$, there is $\Lambda_{m \sigma}>0$ such that problem (2.1) has at least m pairs of solutions $u_{\lambda}$ with $0<J_{\lambda}\left(u_{\lambda}\right) \leq \sigma \lambda^{1-\frac{N}{2}}$ whenever $\lambda \geq \Lambda_{m \sigma}$.

Remark 2.3 We should point out that Theorem 2.3 is different from the previous results of $[9,10]$ in three main directions:

(i) $A(x) \not \equiv 0$. There exist many functions $h(x, t)$ satisfying condition $(\mathrm{H})$, for example, $h(x, t)=P(x)|t|^{p-2} t$, where $P(x)$ is a positive and bounded function.

(ii) Other potentials $V(x)$ guaranteeing compactness of the embedding from $E_{\lambda} \hookrightarrow L^{p}\left(\mathbb{R}^{N}\right)$ can also be used in this paper. For example, (1) $V(x) \in C\left(\mathbb{R}^{N}, \mathbb{R}\right)$ and $\liminf _{|x| \rightarrow \infty} V(x)>V\left(x_{0}\right)=\min _{x \in \mathbb{R}^{N}} V=0$; (2) $V(x) \in C\left(\mathbb{R}^{N}, \mathbb{R}\right)$ with periodic function (or bounded function) and $V\left(x_{0}\right)=\min _{x \in \mathbb{R}^{N}} V(x)=0$.

(iii) We use Lions' second concentration compactness principle and concentration compactness principle at infinity to prove that the $(P S)$ condition holds, which is different from methods used in $[9,10]$.

\section{$3(P S)_{c}$ Condition}

Recall that we say that a sequence $\left(u_{n}\right)$ is a $(P S)$ sequence at level $c\left((P S)_{c}\right.$-sequence, for short) if $\Phi_{\lambda}\left(u_{n}\right) \rightarrow c$ and $\Phi_{\lambda}^{\prime}\left(u_{n}\right) \rightarrow 0 . \Phi_{\lambda}$ is said to satisfy the $(P S)_{c}$ condition if any $(P S)_{c^{-}}$ sequence contains a convergent subsequence.

Lemma 3.1 Let $(\mathrm{V}),(\mathrm{A})$ and $(\mathrm{H})$ be satisfied. Then there exists constant $M(c)$ which is independent of $\lambda \geq 0$ such that $c \geq 0$ and

$$
\limsup _{n \rightarrow \infty}\left\|u_{n}\right\|_{\lambda}^{2} \leq M(c)
$$

Proof Let $\left\{u_{n}\right\}$ be a sequence in $E$ such that

$$
c+o(1)=J\left(u_{n}\right)=\frac{1}{2}\left\|u_{n}\right\|_{\lambda}^{2}-\frac{\lambda}{2^{*}} \int_{\mathbb{R}^{N}}\left|u_{n}\right|^{2^{*}} d x-\frac{\lambda}{2} \int_{\mathbb{R}^{N}} H\left(x,\left|u_{n}\right|^{2}\right) d x,
$$




$$
\begin{aligned}
o(1)\left\|u_{n}\right\|= & \left\langle J^{\prime}\left(u_{n}\right), v\right\rangle \\
= & \operatorname{Re}\left\{\int_{\mathbb{R}^{N}}\left(\nabla_{A} u_{n} \cdot \overline{\nabla_{A} v}+\lambda V(x) u_{n} \bar{v}\right) d x\right. \\
& \left.-\lambda \int_{\mathbb{R}^{N}}\left|u_{n}\right|^{2^{*}-2} u_{n} \bar{v} d x-\lambda \int_{\mathbb{R}^{N}} h\left(x,\left|u_{n}\right|^{2}\right) u_{n} \bar{v} d x\right\} .
\end{aligned}
$$

By (3.1) and (3.2) we have

$$
\begin{aligned}
J_{\lambda}\left(u_{n}\right)-\frac{1}{\mu} J_{\lambda}^{\prime}\left(u_{n}\right) u_{n}= & \left(\frac{1}{2}-\frac{1}{\mu}\right) \int_{\mathbb{R}^{N}}\left(\left|\nabla_{A} u_{n}\right|^{2}+\lambda V(x)\left|u_{n}\right|^{2}\right) d x \\
& +\left(\frac{1}{\mu}-\frac{1}{2^{*}}\right) \lambda \int_{\mathbb{R}^{N}}\left|u_{n}\right|^{2^{*}} d x \\
& +\lambda \int_{\mathbb{R}^{N}}\left(\frac{1}{\mu} h\left(x,\left|u_{n}\right|^{2}\right)\left|u_{n}\right|^{2}-\frac{1}{2} H\left(x,\left|u_{n}\right|^{2}\right)\right) d x
\end{aligned}
$$

On the other hand, condition $\left(\mathrm{h}_{3}\right)$ implies that

$$
\frac{1}{\mu} h\left(x,\left|u_{n}\right|^{2}\right)\left|u_{n}\right|^{2}-\frac{1}{2} H\left(x,\left|u_{n}\right|^{2}\right) \geq 0 .
$$

Thus, it follows from (3.3) that

$$
\left(\frac{1}{2}-\frac{1}{\mu}\right)\left\|u_{n}\right\|_{\lambda}^{2} \leq c+o(1)+\varepsilon_{n}\left\|u_{n}\right\|_{\lambda},
$$

hence for $n$ large enough, we have

$$
\left\|u_{n}\right\|_{\lambda}^{2} \leq \frac{2 \mu}{\mu-2} c
$$

Thus $\left\|u_{n}\right\|_{\lambda}$ is bounded as $n \rightarrow \infty$. Taking the limit in (3.3) shows that $c \geq 0$. This completes the proof of Lemma 3.1.

The main result in this section is the following compactness result.

Lemma 3.2 Suppose that (V), (A) and $(\mathrm{H})$ hold. For any $\lambda \geq 1, J_{\lambda}$ satisfies the $(P S)_{c}$ condition, for all $c \in\left(0, \alpha_{0} \lambda^{1-\frac{N}{2}}\right)$, where $\alpha_{0}=\left(\frac{1}{\mu}-\frac{1}{2^{*}}\right) S^{\frac{N}{2}}$; that is, any $(P S)_{c}$-sequence $\left(u_{n}\right) \subset E_{\lambda}$ has a strongly convergent subsequence in $E_{\lambda}$.

Proof Let $\left\{u_{n}\right\}$ be a $(P S)_{c}$ sequence, by Lemma 3.1, $\left\{u_{n}\right\}$ is bounded in $H_{A}^{1}\left(\mathbb{R}^{N}\right)$. Hence, by diamagnetic inequality, $\left\{\left|u_{n}\right|\right\}$ is bounded in $H_{A}^{1}\left(\mathbb{R}^{N}\right)$. Then, for some subsequence, there is $u \in H_{A}^{1}\left(\mathbb{R}^{N}\right)$ such that $u_{n} \rightarrow u$ in $H_{A}^{1}\left(\mathbb{R}^{N}\right)$. We claim that

$$
\int_{\mathbb{R}^{N}}\left|u_{n}\right|^{2^{*}} d x \rightarrow \int_{\mathbb{R}^{N}}|u|^{2^{*}} d x
$$

In order to prove this claim, we suppose that

$$
|\nabla| u_{n}||^{2} \rightarrow|\nabla| u||^{2}+\sigma \quad \text { and }\left|u_{n}\right|^{2^{*}} \rightarrow|u|^{2^{*}}+v \quad \text { (weak }{ }^{*} \text { sense of measures). }
$$


Using the concentration compactness principle due to Lions ( $c f$. [29, Lemma 1.2]), we obtain a countable index set $I$, sequences $\left\{x_{i}\right\} \subset \mathbb{R}^{N},\left\{\sigma_{i}\right\},\left\{v_{i}\right\} \subset(0, \infty)$ such that

$$
v=\sum_{i \in I} \delta_{x_{i}} \nu_{i}, \quad \sigma \geq \sum_{i \in I} \delta_{x_{i}} \sigma_{i} \quad \text { and } \quad \sigma_{i} \geq S v_{i}^{2 / 2^{*}}
$$

for all $i \in I$, where $\delta_{x_{i}}$ are Dirac measures at $x_{i}$ and $\sigma_{i}, v_{i}$ are constants.

Now, let $x_{i}$ be a singular point of the measures $\sigma$ and $\nu$. We define a function $\phi(x) \in$ $C_{0}^{\infty}\left(\mathbb{R}^{N},[0,1]\right)$ such that $\phi(x)=1$ in $B\left(x_{i}, \varepsilon\right), \phi(x)=0$ in $\mathbb{R}^{N} \backslash B\left(x_{i}, 2 \varepsilon\right)$ and $|\nabla \phi| \leq 2 / \varepsilon$ in $\mathbb{R}^{N}$. Since $\left\{u_{n} \phi\right\}$ is bounded in $H_{A}^{1}\left(\mathbb{R}^{N}\right)$ and $\phi$ takes values in $\mathbb{R}$, a direct calculation shows that

$$
\left\langle J_{\lambda}^{\prime}\left(u_{n}\right), u_{n} \phi\right\rangle \rightarrow 0
$$

and

$$
\overline{\nabla_{A}\left(u_{n} \phi\right)}=i \bar{u}_{n} \nabla \phi+\phi \overline{\nabla_{A} u_{n}}
$$

Therefore,

$$
\begin{aligned}
\int_{\mathbb{R}^{N}}\left|\nabla_{A} u_{n}\right|^{2} \phi d x+\int_{\mathbb{R}^{N}} \lambda V(x)\left|u_{n}\right|^{2} \phi d x \\
=-\operatorname{Re}\left(\int_{\mathbb{R}^{N}} i \bar{u}_{n} \nabla_{A} u_{n} \nabla \phi d x\right)+\lambda \int_{\mathbb{R}^{N}} h\left(x,\left|u_{n}\right|^{2}\right)\left|u_{n}\right|^{2} \phi d x \\
\quad+\lambda \int_{\mathbb{R}^{N}}\left|u_{n}\right|^{2^{*}} \phi d x+o_{n}(1) .
\end{aligned}
$$

By Hölder's inequality, it is not difficult to prove that

$$
\limsup _{n \rightarrow \infty}\left|\operatorname{Re}\left(\int_{\mathbb{R}^{N}} i \bar{u}_{n} \nabla_{A} u_{n} \nabla \phi d x\right)\right|=0 .
$$

In this way, it follows that

$$
\int_{\mathbb{R}^{N}}\left|\nabla_{A} u_{n}\right|^{2} \phi d x \leq \lambda \int_{\mathbb{R}^{N}} h\left(x,\left|u_{n}\right|^{2}\right)\left|u_{n}\right|^{2} \phi d x+\lambda \int_{\mathbb{R}^{N}}\left|u_{n}\right|^{2^{*}} \phi d x+o_{n}(1) .
$$

Consequently, using the fact that $u_{n} \rightarrow u$ in $L_{\text {loc }}^{s}\left(\mathbb{R}^{N}\right), 1 \leq s<2^{*}$ and $\phi$ has compact support, we can let $n \rightarrow \infty$ in the last inequality to obtain

$$
\int_{\mathbb{R}^{N}} \phi d \sigma \leq \lambda \int_{\mathbb{R}^{N}} \phi d \nu
$$

Letting $\varepsilon \rightarrow 0$, we obtain $\sigma_{i} \leq \lambda v_{i}$. Combining this with (3.5), we obtain $v_{i} \geq \lambda^{-1} S v_{i}^{\frac{2}{2^{*}}}$. This result implies that

$$
\text { (I) } v_{i}=0 \quad \text { or } \quad \text { (II) } \quad v_{i} \geq\left(\lambda^{-1} S\right)^{\frac{N}{2}} \text {. }
$$

To obtain the possible concentration of mass at infinity, we will use the concentration compactness principle at infinity [30]. Similarly, we define a cut-off function $\phi_{R} \in$ 
$C_{0}^{\infty}\left(\mathbb{R}^{N},[0,1]\right)$ such that $\phi_{R}(x)=0$ on $|x|<R$ and $\phi_{R}(x)=1$ on $|x|>R+1$. Note that $\left\{u_{n} \phi_{R}\right\}$ is bounded in $H_{A}^{1}\left(\mathbb{R}^{N}\right)$ and $\phi$ takes values in $\mathbb{R}$. A direct calculation shows that $\left\langle J_{\lambda}^{\prime}\left(u_{n}\right), u_{n} \phi_{R}\right\rangle \rightarrow 0$, this fact implies that

$$
\begin{aligned}
\int_{\mathbb{R}^{N}}\left|\nabla_{A} u_{n}\right|^{2} \phi_{R} d x+\int_{\mathbb{R}^{N}} \lambda V(x)\left|u_{n}\right|^{2} \phi_{R} d x \\
=-\operatorname{Re}\left(\int_{\mathbb{R}^{N}} i \bar{u}_{n} \nabla_{A} u_{n} \nabla \phi_{R} d x\right)+\lambda \int_{\mathbb{R}^{N}} h\left(x,\left|u_{n}\right|^{2}\right)\left|u_{n}\right|^{2} \phi_{R} d x \\
\quad+\lambda \int_{\mathbb{R}^{N}}\left|u_{n}\right|^{2^{*}} \phi_{R} d x+o_{n}(1) .
\end{aligned}
$$

It is easy to prove that

$$
-\lim _{R \rightarrow \infty} \lim _{n \rightarrow \infty} \operatorname{Re}\left(\int_{\mathbb{R}^{N}} i \bar{u}_{n} \nabla_{A} u_{n} \nabla \phi_{R} d x\right)=0
$$

Letting $R \rightarrow \infty$, we obtain $\sigma_{\infty} \leq \lambda v_{\infty}$. Thus $v_{\infty} \geq \lambda^{-1} S v_{\infty}^{\frac{2}{2^{*}}}$. This result implies that

$$
\text { (III) } v_{\infty}=0 \text { or (IV) } v_{\infty} \geq\left(\lambda^{-1} S\right)^{\frac{N}{2}} .
$$

Next, we claim that (II) and (IV) cannot occur. If case (IV) holds for some $i \in I$, then by condition $(\mathrm{H})$ we have

$$
\begin{aligned}
c= & \lim _{n \rightarrow \infty}\left(J_{\lambda}\left(u_{n}\right)-\frac{1}{\mu}\left\langle J_{\lambda}^{\prime}\left(u_{n}\right), u_{n}\right\rangle\right) \\
\geq & \left(\frac{1}{2}-\frac{1}{\mu}\right) \int_{\mathbb{R}^{N}}\left(\left|\nabla_{A} u_{n}\right|^{2}+\lambda V(x)\left|u_{n}\right|^{2}\right) d x+\left(\frac{1}{\mu}-\frac{1}{2^{*}}\right) \lambda \int_{\mathbb{R}^{N}}\left|u_{n}\right|^{2^{*}} d x \\
& +\lambda \int_{\mathbb{R}^{N}}\left(\frac{1}{\mu} h\left(x,\left|u_{n}\right|^{2}\right)\left|u_{n}\right|^{2}-\frac{1}{2} H\left(x,\left|u_{n}\right|^{2}\right)\right) d x \\
\geq & \left(\frac{1}{\mu}-\frac{1}{2^{*}}\right) \lambda \int_{\mathbb{R}^{N}}\left|u_{n}\right|^{2^{*}} d x \geq\left(\frac{1}{\mu}-\frac{1}{2^{*}}\right) \lambda \int_{\mathbb{R}^{N}}\left|u_{n}\right|^{2^{*}} \phi_{R} d x \\
= & \left(\frac{1}{\mu}-\frac{1}{2^{*}}\right) \lambda v_{\infty} \geq \alpha_{0} \lambda^{1-\frac{N}{2}} \quad \text { as } R \rightarrow \infty,
\end{aligned}
$$

where $\alpha_{0}=\left(\frac{1}{\mu}-\frac{1}{2^{*}}\right) S^{\frac{N}{2}}$. This is impossible. Consequently, $v_{i}=0$ for all $i \in I$. Similarly, if case (II) holds for some $i \in I$, then by condition (H) we have

$$
\begin{aligned}
c & =\lim _{n \rightarrow \infty}\left(J_{\lambda}\left(u_{n}\right)-\frac{1}{\mu}\left\langle J_{\lambda}^{\prime}\left(u_{n}\right), u_{n}\right\rangle\right) \\
& \geq\left(\frac{1}{\mu}-\frac{1}{2^{*}}\right) \lambda \int_{\mathbb{R}^{N}}\left|u_{n}\right|^{2^{*}} d x \geq\left(\frac{1}{\mu}-\frac{1}{2^{*}}\right) \lambda \int_{\mathbb{R}^{N}}\left|u_{n}\right|^{2^{*}} \phi d x \\
& =\left(\frac{1}{\mu}-\frac{1}{2^{*}}\right) \lambda \nu \geq \alpha_{0} \lambda^{1-\frac{N}{2}} \quad \text { as } \varepsilon \rightarrow 0,
\end{aligned}
$$

which leads to a contradiction. Thus, we must have that (II) cannot occur for each $i$. Thus limit (3.4) holds. 
Thus, from the Brezis-Lieb lemma [31], we have

$$
\begin{aligned}
o(1)\left\|u_{n}\right\|_{\lambda} & =\left\langle J_{\lambda}^{\prime}\left(u_{n}\right), u_{n}\right\rangle=\left\|u_{n}\right\|_{\lambda}^{2}-\lambda \int_{\mathbb{R}^{N}}\left|u_{n}\right|^{2^{*}} d x-\lambda \int_{\mathbb{R}^{N}} H\left(x,\left|u_{n}\right|^{2}\right) d x \\
& =\left\|u_{n}-u\right\|_{\lambda}^{2}+\|u\|_{\lambda}^{2}-\lambda \int_{\mathbb{R}^{N}}|u|^{2^{*}} d x-\lambda \int_{\mathbb{R}^{N}} H\left(x,|u|^{2}\right) d x \\
& =\left\|u_{n}-u\right\|_{\lambda}^{2}+o(1)\|u\|_{\lambda},
\end{aligned}
$$

here we use $J_{\lambda}^{\prime}(u)=0$. Thus we prove that $\left\{u_{n}\right\}$ strongly converges to $u$ in $H_{A}^{1}\left(\mathbb{R}^{N}\right)$. This completes the proof of Lemma 3.2.

\section{Proof of Theorem 2.1}

In the following, we always consider $\lambda \geq 1$. By assumptions (V), (A) and (H), one can see that $J_{\lambda}(u)$ has mountain pass geometry.

Lemma 4.1 Assume that (V), (A) and (H) hold. There exist $\alpha_{\lambda}, \rho_{\lambda}>0$ such that $J_{\lambda}(u)>0$ if $u \in B_{\rho_{\lambda}} \backslash\{0\}$ and $J_{\lambda}(u) \geq \alpha_{\lambda}$ if $u \in \partial B_{\rho_{\lambda}}$, where $B_{\rho_{\lambda}}=\left\{u \in E:\|u\|_{\lambda} \leq \rho_{\lambda}\right\}$.

Proof By condition $(\mathrm{H})$, for $\delta \leq\left(4 \lambda c_{s}\right)^{-1}$, there is $C_{\delta}>0$ such that

$$
\frac{1}{2^{*}} \int_{\mathbb{R}^{N}}|u|^{2^{*}} d x+\frac{1}{2} \int_{\mathbb{R}^{N}} H\left(x,|u|^{2}\right) d x \leq \delta|u|_{2}^{2}+C_{\delta}|u|_{2^{*}}^{2^{*}}
$$

So, from (A) and (V) it follows that

$$
\begin{aligned}
J_{\lambda}(u) & \geq \frac{1}{2}\|u\|_{\lambda}^{2}-\lambda \delta|u|_{2}^{2}-\lambda C_{\delta}|u|_{2^{*}}^{2^{*}} \\
& \geq \frac{1}{4}\|u\|_{\lambda}^{2}-\lambda C_{\delta}|u|_{2^{*}}^{2^{*}} .
\end{aligned}
$$

By (2.2) and $2 *>2$, we know that the conclusion of Lemma 4.1 holds. This completes the proof of Lemma 4.1 .

Lemma 4.2 Under the assumption of Lemma 4.1, for any finite-dimensional subspace $F \subset$ $E_{\lambda}$,

$$
J_{\lambda}(u) \rightarrow-\infty \quad \text { as } u \in F,\|u\| \rightarrow \infty
$$

Proof Using conditions (A), (V) and (H), we can get

$$
J_{\lambda}(u) \leq \frac{1}{2}\|u\|_{\lambda}^{2}-\lambda a_{0}|u|_{p}^{p}
$$

for all $u \in E$ since all norms in a finite-dimensional space are equivalent and $p>2$. This completes the proof of Lemma 4.2.

Since $J_{\lambda}(u)$ does not satisfy the $(P S)_{c}$ condition for all $c>0$, in the following we will find a special finite-dimensional subspace by which we construct sufficiently small minimax levels. 
The assumption (V) implies that there is $x_{0} \in \mathbb{R}^{N}$ such that $V\left(x_{0}\right)=\min _{x \in \mathbb{R}^{N}} V(x)=0$. Without loss of generality we assume from now on that $x_{0}=0$.

Observe that by $\left(h_{3}\right)$

$$
\frac{\lambda}{2^{*}} \int_{\mathbb{R}^{N}} K(x)|u|^{2^{*}} d x+\frac{\lambda}{2} \int_{\mathbb{R}^{N}} H\left(x,|u|^{2}\right) d x \geq a_{0} \lambda \int_{\mathbb{R}^{N}}|u|^{p} d x .
$$

Define the function $I_{\lambda} \in C^{1}\left(E_{\lambda}, \mathbb{R}\right)$ by

$$
I_{\lambda}(u):=\frac{1}{2} \int_{\mathbb{R}^{N}}\left(\left|\nabla_{A} u\right|^{2}+\lambda V(x)|u|^{2}\right) d x-a_{0} \lambda \int_{\mathbb{R}^{N}}|u|^{p} d x .
$$

Then $J_{\lambda}(u) \leq I_{\lambda}(u)$ for all $u \in E$, and it suffices to construct small minimax levels for $I_{\lambda}$.

Note that

$$
\inf \left\{\int_{\mathbb{R}^{N}}|\nabla \phi|^{2} d x: \phi \in C_{0}^{\infty}\left(\mathbb{R}^{N}\right),|\phi|_{2}=1\right\}=0 .
$$

For any $\delta>0$, one can choose $\phi_{\delta} \in C_{0}^{\infty}\left(\mathbb{R}^{N}\right)$ with $\left|\phi_{\delta}\right|_{2}=1$ and $\operatorname{supp} \phi_{\delta} \subset B_{r_{\delta}}(0)$ so that $\left|\nabla \phi_{\delta}\right|_{2}^{2}<\delta$. Set

$$
f_{\lambda}=\phi_{\delta}\left(\lambda^{\frac{1}{2}} x\right)
$$

then

$$
\operatorname{supp} f_{\lambda} \subset B_{\lambda^{-\frac{1}{2}} r_{\delta}}(0)
$$

Thus, for $t \geq 0$, we have

$$
\begin{aligned}
I_{\lambda}\left(t f_{\lambda}\right) & =\frac{t^{2}}{2} \int_{\mathbb{R}^{N}}\left(\left|\nabla_{A} f_{\lambda}\right|^{2}+\lambda V(x)\left|f_{\lambda}\right|^{2}\right) d x-t^{p} a_{0} \lambda \int_{\mathbb{R}^{N}}\left|f_{\lambda}\right|^{p} d x \\
& =\lambda^{1-\frac{N}{2}}\left(\frac{t^{2}}{2} \int_{\mathbb{R}^{N}}\left(\left|\nabla_{A} \phi_{\delta}\right|^{2}+V\left(\lambda^{-\frac{1}{2}} x\right)\left|\phi_{\delta}\right|^{2}\right) d x-t^{p} a_{0} \int_{\mathbb{R}^{N}}\left|\phi_{\delta}\right|^{p} d x\right) \\
& =\lambda^{1-\frac{N}{2}} \Psi_{\lambda}\left(t \phi_{\delta}\right),
\end{aligned}
$$

where $\Psi_{\lambda} \in C^{1}\left(E_{\lambda}, \mathbb{R}\right)$ defined by

$$
\Psi_{\lambda}(u):=\frac{1}{2} \int_{\mathbb{R}^{N}}\left(\left|\nabla_{A} u\right|^{2}+V\left(\lambda^{-\frac{1}{2}} x\right)|u|^{2}\right) d x-a_{0} \int_{\mathbb{R}^{N}}|u|^{p} d x .
$$

Obviously,

$$
\max _{t \geq 0} \Psi_{\lambda}\left(t \phi_{\delta}\right)=\frac{p-2}{2 p\left(p a_{0}\right)^{\frac{2}{p-2}}}\left[\int_{\mathbb{R}^{N}}\left(\left|\nabla_{A} \phi_{\delta}\right|^{2}+V\left(\lambda^{-\frac{1}{2}} x\right)\left|\phi_{\delta}\right|^{2}\right) d x\right]^{\frac{p}{p-2}} .
$$

On the one hand, since $V(0)=0$ and note that $\operatorname{supp} \phi_{\delta} \subset B_{r_{\delta}}(0)$, there is $\Lambda_{\delta_{1}}>0$ such that

$$
V\left(\lambda^{-\frac{1}{2}} x\right) \leq \frac{\delta}{\left|\phi_{\delta}\right|_{2}^{2}} \quad \text { for all }|x| \leq r_{\delta} \text { and } \lambda \geq \Lambda_{\delta_{1}}
$$


On the other hand, by Hölder's inequality, we have

$$
\int_{\mathbb{R}^{N}}\left|\nabla_{A} \phi_{\delta}\right|^{2} d x \leq \int_{\mathbb{R}^{N}}\left(2\left|\nabla \phi_{\delta}\right|^{2}+2\left|A\left(\lambda^{-\frac{1}{2}} x\right) \phi_{\delta}\right|^{2}\right) d x .
$$

Since $A(x)$ is continuous on $\mathbb{R}^{N}$ and $A(0)=0$, there exists $\Lambda_{\delta_{2}}>0$ such that

$$
\left|A\left(\lambda^{-\frac{1}{2}} x\right)\right| \leq \sqrt{\frac{\delta}{\left|\phi_{\delta}\right|_{2}^{2}}} \quad \text { for all }|x| \leq r_{\delta} \text { and } \lambda \geq \Lambda_{\delta_{2}}
$$

Without loss of generality, we take $\Lambda_{\delta}:=\left\{\Lambda_{\delta_{1}}, \Lambda_{\delta_{2}}\right\}$. So, by (4.2) and (4.3) we can get

$$
\max _{t \geq 0} \Psi_{\lambda}\left(t \phi_{\delta}\right) \leq \frac{p-2}{2 p\left(p a_{0}\right)^{\frac{2}{p-2}}}(5 \delta)^{\frac{p}{p-2}}
$$

Therefore, for all $\lambda \geq \Lambda_{\delta}$,

$$
\max _{t \geq 0} J_{\lambda}\left(t \phi_{\delta}\right) \leq \frac{p-2}{2 p\left(p a_{0}\right)^{\frac{2}{p-2}}}(5 \delta)^{\frac{p}{p-2}} \lambda^{1-\frac{N}{2}}
$$

Thus we have the following lemma.

Lemma 4.3 Under the assumption of Lemma 4.1, for any $\sigma>0$, there exists $\Lambda_{\sigma}>0$ such that for each $\lambda \geq \Lambda_{\sigma}$, there is $\hat{f}_{\lambda} \in E_{\lambda}$ with $\left\|\hat{f}_{\lambda}\right\|>\rho_{\lambda}, J_{\lambda}\left(\hat{f}_{\lambda}\right) \leq 0$ and

$$
\max _{t \in[0,1]} J_{\lambda}\left(t \hat{f}_{\lambda}\right) \leq \sigma \lambda^{1-\frac{N}{2}}
$$

Proof Choose $\delta>0$ so small that

$$
\frac{p-2}{2 p\left(p a_{0}\right)^{\frac{2}{p-2}}}(5 \delta)^{\frac{p}{p-2}} \leq \sigma
$$

and let $f_{\lambda} \in E$ be the function defined by (4.1). Taking $\Lambda_{\sigma}=\Lambda_{\delta}$. Let $\hat{t}_{\lambda}>0$ be such that $\hat{t}_{\lambda}\left\|f_{\lambda}\right\|_{\lambda}>\rho_{\lambda}$ and $J_{\lambda}\left(t f_{\lambda}\right) \leq 0$ for all $t \geq \hat{t}_{\lambda}$. By (4.3), let $\hat{f}_{\lambda}=\hat{t}_{\lambda} f_{\lambda}$; we know that the conclusion of Lemma 4.3 holds.

For any $m^{*} \in N$, one can choose $m^{*}$ functions $\phi_{\delta}^{i} \in C_{0}^{\infty}\left(\mathbb{R}^{N}\right)$ such that $\operatorname{supp} \phi_{\delta}^{i} \cap \operatorname{supp} \phi_{\delta}^{k}=$ $\emptyset, i \neq k,\left|\phi_{\delta}^{i}\right|_{p}=1$ and $\left|\nabla \phi_{\delta}^{i}\right|_{2}^{2}<\delta$. Let $r_{\delta}^{m^{*}}>0$ be such that $\operatorname{supp} \phi_{\delta}^{i} \subset B_{r_{\delta}}^{i}(0)$ for $i=1,2, \ldots, m^{*}$. Set

$$
f_{\lambda}^{i}(x)=\phi_{\delta}^{i}\left(\lambda^{\frac{1}{2}} x\right) \quad \text { for } j=1,2, \ldots, m^{*}
$$

and

$$
H_{\lambda \delta}^{m^{*}}=\operatorname{span}\left\{f_{\lambda}^{1}, f_{\lambda}^{2}, \ldots, f_{\lambda}^{m^{*}}\right\}
$$

Observe that for each $u=\sum_{i=1}^{m^{*}} c_{i} f_{\lambda}^{i} \in H_{\lambda \delta}^{m^{*}}$,

$$
\int_{\mathbb{R}^{N}}\left|\nabla_{A} u\right|^{2} d x=\sum_{i=1}^{m^{*}}\left|c_{i}\right|^{2} \int_{\mathbb{R}^{N}}\left|\nabla_{A} f_{\lambda}^{i}\right|^{2} d x
$$




$$
\begin{aligned}
& \int_{\mathbb{R}^{N}} V(x)|u|^{2} d x=\sum_{i=1}^{m^{*}}\left|c_{i}\right|^{2} \int_{\mathbb{R}^{N}} V(x)\left|f_{\lambda}^{i}\right|^{2} d x, \\
& \frac{1}{2^{*}} \int_{\mathbb{R}^{N}}|u|^{2^{*}} d x=\frac{1}{2^{*}} \sum_{i=1}^{m^{*}}\left|c_{i}\right|^{2^{*}} \int_{\mathbb{R}^{N}}|u|^{2^{*}} d x
\end{aligned}
$$

and

$$
\frac{1}{2} \int_{\mathbb{R}^{N}} H\left(x,|u|^{2}\right) d x=\frac{1}{2} \sum_{i=1}^{m^{*}} \int_{\mathbb{R}^{N}} H\left(x,\left|c_{i} f_{\lambda}^{i}\right|^{2}\right) d x .
$$

Thus

$$
J_{\lambda}(u)=\sum_{i=1}^{m^{*}} J_{\lambda}\left(c_{i} f_{\lambda}^{i}\right)
$$

and as before

$$
J_{\lambda}\left(c_{i} f_{\lambda}^{i}\right) \leq \lambda^{1-\frac{N}{2}} \Psi\left(\left|c_{i}\right| f_{\lambda}^{i}\right) .
$$

Set

$$
\beta_{\delta}:=\max \left\{\left|\phi_{\delta}^{i}\right|_{2}^{2}: j=1,2, \ldots, m^{*}\right\}
$$

and choose $\Lambda_{m^{*} \delta}>0$ so that

$$
V\left(\lambda^{1-\frac{N}{2}} x\right) \leq \frac{\delta}{\beta_{\delta}} \quad \text { for all }|x| \leq r_{\delta}^{m^{*}} \text { and } \lambda \geq \Lambda_{m^{*} \delta}
$$

As before, we can obtain the following:

$$
\max _{u \in H_{\lambda \delta}^{m^{*}}} J_{\lambda}(u) \leq \frac{m^{*}(p-2)}{2 p\left(p a_{0}\right)^{\frac{2}{p-2}}}(5 \delta)^{\frac{p}{p-2}} \lambda^{1-\frac{N}{2}}
$$

for all $\lambda \geq \Lambda_{m^{*} \delta}$.

Using this estimate we have the following.

Lemma 4.4 Under the assumption of Lemma 4.1, for any $m^{*} \in \mathbb{N}$ and $\sigma>0$, there exists $\Lambda_{m^{*} \sigma}>0$ such that for each $\lambda \geq \Lambda_{m^{*} \sigma}$, there exists an $m^{*}$-dimensional subspace $F_{\lambda m^{*}}$ satisfying

$$
\max _{u \in F_{\lambda \delta}} J_{\lambda}(u) \leq \sigma \lambda^{1-\frac{N}{2}} .
$$

Proof Choose $\delta>0$ so small that

$$
\frac{m^{*}(p-2)}{2 p\left(p a_{0}\right)^{\frac{2}{p-2}}}(5 \delta)^{\frac{p}{p-2}} \leq \sigma
$$

and take $F_{\lambda \delta}=H_{\lambda \delta}^{m^{*}}$. By (4.5), we know that the conclusion of Lemma 4.4 holds. 
We now establish the existence and multiplicity results.

Proof of Theorem 2.1 Using Lemma 4.3, we choose $\Lambda_{\sigma}>0$ and define for $\lambda \geq \Lambda_{\sigma}$ the minimax value

$$
c_{\lambda}:=\inf _{\gamma \in \Gamma_{\lambda}} \max _{t \in[0,1]} J_{\lambda}\left(t \hat{f}_{\lambda}\right)
$$

where

$$
\Gamma_{\lambda}:=\left\{\gamma \in C([0,1], E): \gamma(0)=0 \text { and } \gamma(1)=\hat{f}_{\lambda}\right\}
$$

By Lemma 4.1, we have $\alpha_{\lambda} \leq c_{\lambda} \leq \sigma \lambda^{1-\frac{N}{2}}$. In virtue of Lemma 3.2, we know that $J_{\lambda}$ satisfies the $(P S)_{c_{\lambda}}$ condition, there is $u_{\lambda} \in E$ such that $J_{\lambda}^{\prime}\left(u_{\lambda}\right)=0$ and $J_{\lambda}\left(u_{\lambda}\right)=c_{\lambda}$, hence the existence is proved.

Denote the set of all symmetric (in the sense that $-Z=Z$ ) and closed subsets of $E$ by $\Sigma$ for each $Z \in \Sigma$. Let gen $(Z)$ be the Krasnoselski genus and

$$
i(Z):=\min _{h \in \Gamma_{m^{*}}} \operatorname{gen}\left(h(Z) \cap \partial B_{\rho_{\lambda}}\right),
$$

where $\Gamma_{m^{*}}$ is the set of all odd homeomorphisms $h \in C(E, E)$ and $\rho_{\lambda}$ is the number from Lemma 4.1. Then $i$ is a version of Benci's pseudo-index [32]. Let

$$
c_{\lambda i}:=\inf _{i(Z) \geq i} \sup _{u \in Z} J_{\lambda}(u), \quad 1 \leq i \leq m^{*} .
$$

Since $J_{\lambda}(u) \geq \alpha_{\lambda}$ for all $u \in \partial B_{\rho \lambda}^{+}$and since $i\left(F_{\lambda m^{*}}\right)=\operatorname{dim} F_{\lambda m^{*}}=m^{*}$,

$$
\alpha_{\lambda} \leq c_{\lambda 1} \leq \cdots \leq c_{\lambda m^{*}} \leq \sup _{u \in H_{\lambda m^{*}}} J_{\lambda}(u) \leq \sigma \lambda^{1-\frac{N}{2}}
$$

It follows from Lemma 3.2 that $J_{\lambda}$ satisfies the $(P S)_{c_{\lambda}}$ condition at all levels $c_{i}$. By the usual critical point theory, all $c_{i}$ are critical levels and $J_{\lambda}$ has at least $m^{*}$ pairs of nontrivial critical points.

Competing interests

The authors declare that they have no competing interests.

Authors' contributions

SL carried out the theoretical studies, participated in the sequence alignment and drafted the manuscript. YS participated in the design of the study and performed the statistical analysis. All authors read and approved the final manuscript.

\section{Author details}

${ }^{1}$ College of Mathematics, Changchun Normal University, Changchun, Jilin 130032, P.R. China. ${ }^{2}$ Key Laboratory of Symbolic Computation and Knowledge Engineering of Ministry of Education, Jilin University, Changchun, 130012 P.R. China. ${ }^{3}$ Scientific Research Department, Changchun Normal University, Changchun, Jilin 130032, P.R. China.

\section{Acknowledgements}

The authors would like to appreciate the referees for their precious comments and suggestions about the original manuscript. The authors are supported by the National Natural Science Foundation of China (Grant No. 11301038), Research Foundation during the 12th Five-Year Plan Period of Department of Education of Jilin Province, China (Grant (2013) No. 252), Youth Foundation for Science and Technology Department of Jilin Province (20130522100JH), the open project program of Key Laboratory of Symbolic Computation and Knowledge Engineering of Ministry of Education, Jilin University (Grant No. 93K172013K03). 


\section{References}

1. Byeon, J, Wang, ZQ: Standing waves with a critical frequency for nonlinear Schrödinger equations. Arch. Ration. Mech. Anal. 165, 295-316 (2002)

2. Ambrosetti, A, Badiale, M, Cingolani, S: Semiclassical states of nonlinear Schrödinger equations. Arch. Ration. Mech. Anal. 140, 285-300 (1997)

3. Ambrosetti, A, Malchiodi, A, Secchi, S: Multiplicity results for some nonlinear Schrödinger equations with potentials. Arch. Ration. Mech. Anal. 159, 253-271 (2001)

4. Cingolani, S: Semiclassical stationary states of nonlinear Schrödinger equation with external magnetic field. J. Differ. Equ. 188, 52-79 (2003)

5. Cingolani, S, Lazzo, M: Multiple positive solutions to nonlinear Schrödinger equations with competing potential functions. J. Differ. Equ. 160, 118-138 (2000)

6. Cingolani, S, Nolasco, M: Multi-peaks periodic semiclassical states for a class of nonlinear Schrödinger equations. Proc R. Soc. Edinb. 128, 1249-1260 (1998)

7. Del Pino, M, Felmer, P: Semi-classical states for nonlinear Schrödinger equations. Ann. Inst. Henri Poincaré 15, 127-149 (1998)

8. Del Pino, M, Felmer, P: Multi-peak bound states for nonlinear Schrödinger equations. J. Funct. Anal. 149, 245-265 (1997)

9. Ding, YH, Lin, FH: Solutions of perturbed Schrödinger equations with critical nonlinearity. Calc. Var. Partial Differ. Equ. 30, 231-249 (2007)

10. Ding, YH, Wei, JC: Semiclassical states for nonlinear Schrödinger equations with sign-changing potentials. J. Funct. Anal. 251, 546-572 (2007)

11. Floer, A, Weinstein, A: Nonspreading wave packets for the cubic Schrödinger equation with a bounded potential. J. Funct. Anal. 69, 397-408 (1986)

12. Oh, Y-G: On positive multi-lump bound states of nonlinear Schrödinger equations under multiple well potential. Commun. Math. Phys. 131, 223-253 (1990)

13. Wang, X: On concentration of positive bound states of nonlinear Schrödinger equations. Commun. Math. Phys. 153 229-244 (1993)

14. Kang, $X$, Wei, J: On interacting bumps of semi-classical states of nonlinear Schrödinger equations. Adv. Differ. Equ. 5, 899-928 (2000)

15. Brezis, H, Nirenberg, L: Positive solutions of nonlinear elliptic equations involving critical exponents. Commun. Pure Appl. Math. 34, 437-477 (1983)

16. Esteban, M, Lions, PL: Stationary solutions of nonlinear Schrödinger equations with an external magnetic field. In: Partial Differential Equations and the Calculus of Variations. Essays in Honor of Ennio De Giorgi, pp. 369-408 (1989)

17. Arioli, G, Szulkin, A: A semilinear Schrödinger equation in the presence of a magnetic field. Arch. Ration. Mech. Anal. $170,277-295(2003)$

18. Kurata, K: Existence and semi-classical limit of the least energy solution to a nonlinear Schrödinger equation with electromagnetic fields. Nonlinear Anal. 41, 763-778 (2000)

19. Cingolani, S, Secchi, S: Semiclassical limit for nonlinear Schrödinger equation with electromagnetic fields. J. Math. Anal. Appl. 275, 108-130 (2002)

20. Wang, F: On an electromagnetic Schrödinger equation with critical growth. Nonlinear Anal. 69, 4088-4098 (2008)

21. Chabrowski, J, Szulkin, A: On the Schrödinger equation involving a critical Sobolev exponent and magnetic field. Topol. Methods Nonlinear Anal. 25, 3-21 (2005)

22. Han, P: Solutions for singular critical growth Schrödinger equation with magnetic field. Port. Math. 63, 37-45 (2006)

23. Barile, S, Cingolani, S, Secchi, S: Single-peaks for a magnetic Schrödinger equation with critical growth. Adv. Differ. Equ. 11, 1135-1166 (2010)

24. Cao, D, Noussair, ES: Multi-bump standing waves with a critical frequency for nonlinear Schrödinger equations. J. Differ. Equ. 203, 292-312 (2004)

25. Cao, D, Tang, Z: Existence and uniqueness of multi-bump bound states of nonlinear Schrödinger equations with electromagnetic fields. J. Differ. Equ. 222, 381-424 (2006)

26. Tang, Z: On the least energy solutions of nonlinear Schrödinger equations with electromagnetic fields. Comput. Math. Appl. 54, 627-637 (2007)

27. Tang, Z: Multi-bump bound states of nonlinear Schrödinger equations with electromagnetic fields and critical frequency. J. Differ. Equ. 245, 2723-2748 (2008)

28. Willem, M: Minimax Theorems. Birkhäuser Boston, Boston (1996)

29. Lions, PL: The concentration compactness principle in the calculus of variations. The locally compact case. Part I. Ann. Inst. Henri Poincaré, Anal. Non Linéaire 1, 109-145 (1984)

30. Chabrowski, J: Concentration-compactness principle at infinity and semilinear elliptic equations involving critical and subcritical Sobolev exponents. Calc. Var. 3, 493-512 (1995)

31. Brezis, H, Lieb, E: A relation between pointwise convergence of functions and convergence of functionals. Proc. Am. Math. Soc. 88, 486-490 (1983)

32. Benci, V: On critical point theory of indefinite functionals in the presence of symmetries. Trans. Am. Math. Soc. 274, 533-572 (1982)

doi:10.1186/s13661-014-0240-

Cite this article as: Liang and Song: Multiplicity of solutions of perturbed Schrödinger equation with electromagnetic fields and critical nonlinearity in $\mathbb{R}^{N}$. Boundary Value Problems 2014 2014:240. 